\title{
Breastfeeding promotion interventions and breastfeeding practices: a systematic review
}

\author{
Sarah Haroon', Jai K Das ${ }^{1}$, Rehana A Salam¹, Aamer Imdad ${ }^{1}$, Zulfiqar A Bhutta ${ }^{1,2^{*}}$
}

\begin{abstract}
Introduction: Exclusive Breastfeeding (EBF) rates remain low in both low-income and high-income countries despite World Health Organization recommendations for EBF till 6 months. Breastfeeding has been shown to have a protective effect against gastrointestinal infections, among other benefits. Large-scale interventions focusing on educating mothers about breastfeeding have the potential to increase breastfeeding prevalence, especially EBF, up to recommended standards and also to decrease infant morbidity.

Methods: A systematic literature search was conducted for RCTs and quasi-experimental studies comparing breastfeeding education or support to routine care. The effect of interventions was observed for exclusive, predominant, partial and no breastfeeding rates. The time intervals of interest were day $1,<1$ month, and 1 to 5 months. Outcome-specific evidence was graded according to the Child Health Epidemiology Reference Group (CHERG) rules using the adapted Grading of Recommendations, Assessment, Development and Evaluation (GRADE) criteria and recommendations were made from studies in developing countries for inclusion into the Lives Saved Tool (LiST) model.

Results: After reviewing 4600 abstracts, 372 studies were selected for full text screening and 110 of these studies were finally included. Statistically significant increases in EBF rates as a result of breastfeeding promotion interventions were observed: $43 \%$ at day 1, 30\% at $<1$ month, and $90 \%$ at 1-5 months. Rates of 'no breastfeeding' reduced by $32 \%$ at 1 day, 30\% at $<1$ month, and 18\% at 1-5 months. The effect of interventions on the rates of predominant and partial breastfeeding were non-significant.

Conclusion: Breastfeeding education and/or support increased EBF rates and decreased no breastfeeding rates at birth, $<1$ month and 1-5 months. Combined individual and group counseling appeared to be superior to individual or group counseling alone. Interventions in developing countries had a greater impact than those in developed countries.
\end{abstract}

\section{Introduction}

The World Health Organization (WHO) recommends exclusive breastfeeding (EBF) to infants till 6 months of age to achieve optimum growth [1]. Despite this, EBF remains uncommon in most countries, even in countries with high rates of breastfeeding initiation $[2,3]$. In the developing world, one out of every three children is exclusively breastfed for the first six months of life, though considerable variation exists across regions [4]. Recent data shows that the prevalence of EBF in developing countries has increased from 33\% in 1995 to just 39\%

\footnotetext{
* Correspondence: zulfiqar.bhutta@aku.edu

'Division of Women \& Child Health, The Aga Khan University, Karachi 74800, Pakistan

Full list of author information is available at the end of the article
}

in 2010 [5]. These figures were based on 66 countries covering $74 \%$ of the developing world population. The prevalence of EBF increased in almost all regions in the developing world, with a major improvement seen in West and Central Africa where the prevalence doubled from $12 \%$ to $28 \%$, while more modest improvements were observed in South Asia where the increase was from $40 \%$ in 1995 to $45 \%$ in 2010 . A recent WHO report shows that the median coverage of EBF has increased from $26 \%$ in $2000-2005$ to $40 \%$ in $2006-2011$ in the 48 Countdown countries [6].

EBF has protective effects against gastrointestinal infection [1] and the high incidence of morbidity and mortality from gastrointestinal infection in developing countries demands large-scale interventions to increase
C Biomed Central

() 2013 Haroon et al; licensee BioMed Central Ltd. This is an Open Access article distributed under the terms of the Creative Commons Attribution License (http://creativecommons.org/licenses/by/2.0), which permits unrestricted use, distribution, and reproduction in any medium, provided the original work is properly cited. 
breastfeeding prevalence and exclusivity as evidence shows that "no breast feeding" is associated with a significant $165 \%$ increase in diarrhoea incidence in 0-5 month old infants and a $32 \%$ increase in 6-11 month old infants [7].

Lack of knowledge and confidence were found as the main reasons among mothers for less than optimum breastfeeding duration $[8,9]$. Perception of insufficient milk and work outside the home were cited as common reasons for premature weaning or not breast-feeding exclusively $[10,11]$. Pediatricians, nurses, midwives and lay counselors should therefore actively promote and educate, while taking into account mothers' situational limitations.

Strategies that have been successful in increasing breastfeeding rates are the Baby Friendly Hospital Initiative (BFHI) [12], and the use of peer counselors in settings where home deliveries are predominant $[13,14]$. Largescale interventions including the Integrated Management of Childhood Illness (IMCI) program in developing countries, which have shown to improve feeding practices and reduced growth faltering $[15,16]$. Other strategies that have been employed to increase education include mother-to-mother support and contact with lay counselors or trained personnel via home visits $[11,17]$ or telephone-based support $[10,18]$. These interventions may be carried out in a one-to-one counseling session or may occur in a group setting [19-22] or peer support groups [11]. Apart from interactive counseling strategies, largescale awareness programs have also been launched via mass, electronic and print media.

Several reviews on the effect of educational interventions to increase breastfeeding have been conducted. A review by Chapman et al [23] found that peer counselors effectively improved breastfeeding initiation, duration and exclusivity. A recent Cochrane review by Lumbiganon et al [24] found that peer counseling, lactation consultation and formal BF education during pregnancy increased BF duration. The review conducted by Imdad et al [25] concludes that EBF rates rose significantly as a result of educational interventions, with a greater effect observed in developing countries. We in this review have updated the previous review by Imdad et al [25] to include the studies published after the last search date and have in addition expanded it to examine the effect of these interventions beyond EBF to include predominant, partial and no breastfeeding rates. We have reviewed and evaluated the quality of included studies according to the Child Health Epidemiology Reference Group (CHERG) adaptation of Grading of Recommendations, Assessments, Development and Education (GRADE) criteria [26].

\section{Methods}

We searched published literature from PubMed, Medline, Cochrane Library, EMBASE and WHO regional databases to identify studies examining the effects of interventions to promote breastfeeding on breastfeeding rates; exclusive, predominant, partial or no breastfeeding. We used the Medical Subject Heading (MeSH) Terms and keywords in various combinations. No language or date restrictions were employed in the electronic searches. Two authors independently assessed the eligibility using pre-defined inclusion and exclusion criteria and performed data extraction. Any discrepancies between the reviewers in either the decision of inclusion or exclusion of studies or in data extraction were resolved by discussion aimed at reaching consensus. If two or more studies presented data for the same population during the same time period, the most applicable study based on methods and analysis was included in the meta-analyses.

\section{Inclusion criteria}

We selected studies that were either randomized controlled trials (RCTs) or quasi-experimental trials. Studies with community- or facility-based interventions were included. The type of interventions included were those that offered education and/or support given to mothers through counselors (lay counselors and health professionals), and in either individual or group sessions, or a combination of both. All studies where intervention (education/support) was given either in prenatal, postnatal, or combined prenatal and postnatal periods, were included. Studies were included irrespective of the mode of delivery, whether vaginal or cesarean. For non-English articles, we primarily relied on the abstracts but did not translate the entire article into English. If the desired outcome was not present in the abstract, the study was excluded.

\section{Exclusion criteria}

We excluded studies that had before-after study designs, or were cohort and cross-sectional studies. All studies in which interventions were given specifically to preterm/ very preterm babies, low birth weight/very low birth weight babies, babies with prenatal disease, born to drugusing mothers or babies in the Neonatal Intensive Care Unit (NICU) were excluded. Other interventions for promotion of breastfeeding like skin-to-skin contact or delayed pacifier use with the goal of decreasing ambivalence and resistance toward sustained breastfeeding were excluded.

\section{Abstraction, analysis and summary measure}

For the studies that met the final inclusion criteria, double data abstraction was done describing study identifiers and context, study design and limitations, intervention specifics and outcome effects into a standardized abstraction form as detailed in the CHERG Systematic Review Guidelines [26]. Each study was assessed and graded 
according to the CHERG adaptation of the GRADE technique [27].

\section{Quantitative data synthesis}

For any outcome with more than one study, we conducted a meta-analysis using Revman 5.2 [28] and reported the Mantel-Haenszel pooled relative risk (RR) and corresponding 95\% confidence interval (CI). Heterogeneity was assessed by a low $\mathrm{P}$ value (less than 0.1 ) or a large chi-squared statistic relative to its degree of freedom. The $\mathrm{I}^{2}$ values were also examined, and a value greater than $50 \%$ was interpreted as representing substantial and high heterogeneity, where causes were explored and the random effects model used.

Subgroup analyses were also done for studies; group vs. individual counseling, community based interventions vs. facility based interventions, and developing countries vs. developed countries. We summarized the evidence by outcome, including qualitative assessments of study quality and quantitative measures, according to the standard guidelines. A grade of "high", "moderate", "low" and "very low" was used for grading the overall evidence indicating the strength of an effect on specific health outcome according to the CHERG Rules for Evidence Review [26].

\section{Outcomes and definitions}

We have specified breastfeeding outcomes according to the categories of breastfeeding defined by the WHO $[29,30]$. The outcomes of interest included 'EBF', 'Predominant breastfeeding', 'Partial breastfeeding' and 'No breastfeeding' rates at day $1,<1$ month and 1-5 months age.

'EBF' was defined as the child receiving only breast milk (including milk expressed or from a wet nurse) and no other type of milk or solids but could include vitamins, drops of other medicines and oral rehydration therapy (ORT). 'Predominant breastfeeding' was defined as the infant having breast milk as the predominant source of nourishment; however, the infant may also have received liquids (water and water-based drinks, fruit juice), ritual fluids and ORT, drops or syrups (vitamins, minerals and medicines). 'Partial breastfeeding' was defined as giving a baby some breastfeeds, and some artificial feeds, either milk or cereal, or other food. 'No breastfeeding' was defined as infants receiving no breast milk at all.

The time intervals of interest; day $1,<1$ month and 1-5 months, were selected. "Day 1" was intended to refer to the early postpartum period and was extended to include time at hospital discharge provided it occurred at approximately the routine 48 -hours postpartum. Where more than one data point was presented during this time period in a study, the earlier one was selected. The time interval " $<1$ month" included the time beginning from the end of the early postpartum period to 30 days. The time interval "1-5 months" included the beginning of the $2^{\text {nd }}$ month to the end of 6 months. Breastfeeding rates after 6 months were recorded if they were reported in studies. For each of these time intervals, when multiple data for outcomes belonging to the same time interval were presented, the later data point was selected.

We performed subgroup analyses based on the types of counseling. "Individual counseling" was defined as interventions which solely had individual counseling and included one-on-one education or social support via home visits or telephone support. "Group counseling", was defined as interventions with solely group counseling, including education or support sessions, discussions or classes in groups directed at mothers or other family members. "Individual and group counseling" included those studies that used interventions involving both individual and group counseling.

Subgroup analyses were also done based on the level of care. "Community-based interventions" included studies that had interventions conducted solely at the community level, in the form of care given at the home or in community and village centers, or disbursed throughout the community as an awareness program. "Facility-based interventions" was defined as interventions conducted solely at the facility level, including hospitals (such as the BFHI) and outpatient clinics or involving follow-up with facility-based professionals in the form of telephone calls. "Facility- and community-based interventions" included those studies that used interventions that were conducted at both the facility and community level.

Subgroup analyses were also conducted based on the country in which the intervention took place. The World Bank list of economies was used to classify developing (low-income and middle-income) and developed (highincome) countries [31].

\section{Results}

We conducted the search on October 5, 2012 and updated it on November 27, 2012. We screened 4600 titles and abstracts identified through literature searches and contacts with subject area experts. Of these, we reviewed 372 papers and included 110 in our final database (Figure 1). These studies included 63 RCTs and 47 quasi-experimental studies. Of these studies, 78 had individual counseling, 14 had group counseling, and 19 studies had both individual and group counseling. 21 of these studies were done in community, 46 in facility while 43 were both community- and facility-based. 34 studies were conducted in developing countries and 76 in developed countries.

Various educational interventions were employed by the included studies; counseling during home visits was used in 39 studies, peer counseling in 18 studies and peer support groups in three studies. 30 studies used telephonic 


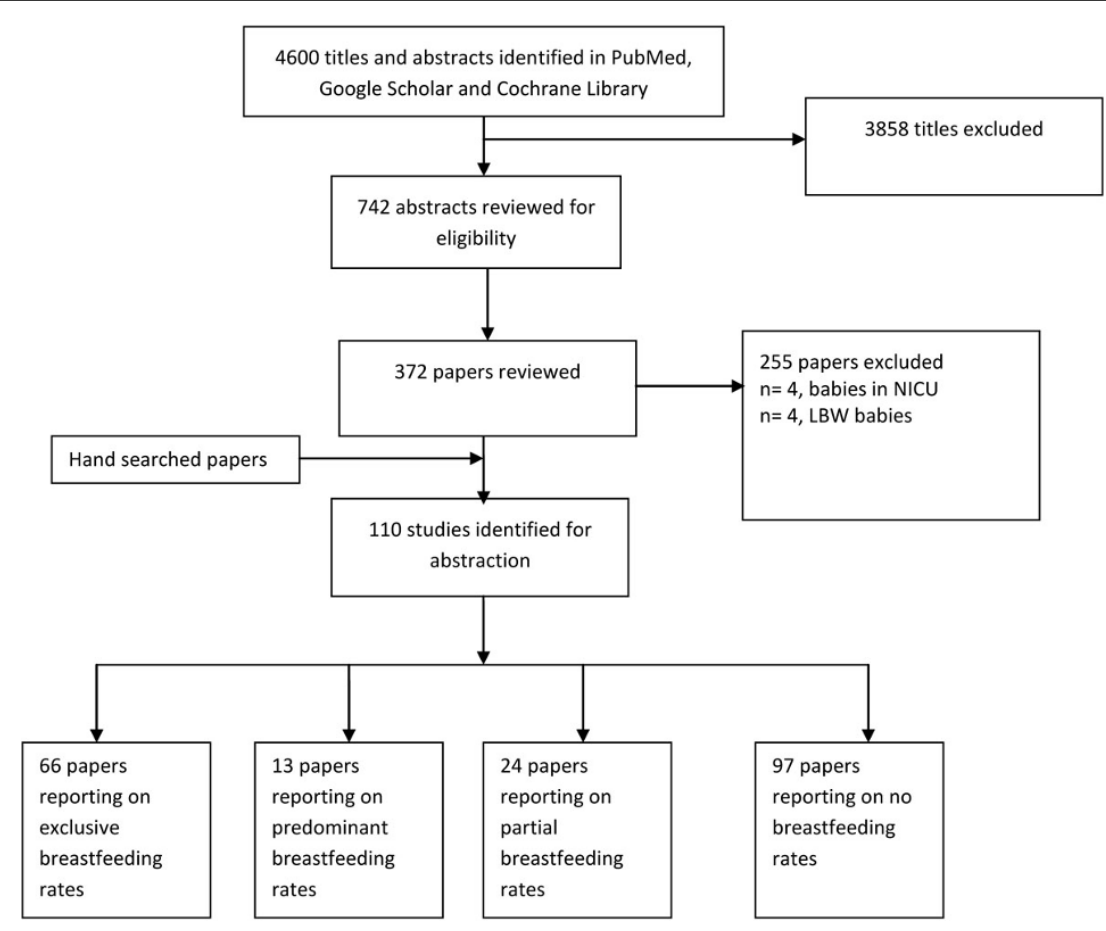

Figure 1 Search strategy flow diagram

counseling and two studies used Internet or softwarebased educational programs. 16 studies used formal educational classes and 15 studies used in-hospital counseling. There were three studies that used counseling of fathers as the primary intervention.

\section{Exclusive breastfeeding rates}

In Table 1, we report the quality assessment of breastfeeding promotion intervention on EBF. A total of 66 studies were included for this outcome [11-14,17-22,32-87]. 27 of 66 studies were conducted in developing countries. Overall, educational interventions significantly increased EBF rates at day 1 by $43 \%$ (RR: 1.43 , 95\% CI: $1.09-1.87$ ), at $<1$ month by $30 \%$ (RR: $1.30,95 \%$ CI: $1.19-1.42$ ) and at $1-5$ months by $90 \%$ (RR: 1.90, 95\% CI: 1.54-2.34) (Figure 2).

At day 1, subgroup analyses showed that individual counseling alone led to a $60 \%$ increase (RR: $1.60,95 \% \mathrm{CI}$ : 1.04-2.48) while the effects of group counseling alone or combined individual and group counseling were nonsignificant. Subgroup analyses for the level of care showed that results were significant only for facilitybased interventions. In developing countries, these interventions led to an increase of $157 \%$ (RR: $2.57,95 \% \mathrm{CI}$ : 1.39-4.77) whereas a non-significant effect was demonstrated in developed countries.

For the $<1$ month interval, subgroup analyses showed that the effects of individual counseling and combined individual and group counseling were significant, with increases of $31 \%$ and $27 \%$ respectively. Facility-based interventions were found to increase EBF rates significantly by $26 \%$ (RR: $1.26,95 \%$ CI: $1.11-1.43$ ) and combined facility and community based interventions showed significant increase of $31 \%$ (RR: 1.31, 95\%CI: 1.14-1.50). The effects were significant for both developing and developed countries at 35\% (RR: 1.35, 95\% CI: $1.15-1.58$ ) and 26\% (RR: $1.26,95 \% \mathrm{CI}: 1.13-1.41$ ) respectively.

At 1-5 months, subgroup analyses showed that both individual and group counseling alone had significant impacts at $90 \%$ (RR: $1.90,95 \%$ CI: $1.54-2.34$ ) and $80 \%$ (RR: 1.80, 95\% CI: 1.18-2.74), respectively. Combined individual and group counseling led to an increase of 101\% (RR: 2.01, 95\% CI: 1.43-2.82), Subgroup analyses for level of care revealed that both community and facility-based care had significant results at 159\% (RR: 2.59 , 95\% CI: 1.80-3.73) and 87\% (RR: 1.87, 95\% CI: 1.26-2.78) respectively and the effect of combined facility- and community-based care was an increase of $47 \%$ (RR: $1.47,95 \%$ CI: 1.08-1.99). Interventions in developing countries led to a significant increase of $188 \%$ (RR: 2.88 , 95\% CI 2.113.93), while the impact was non-significant for developed countries.

\section{Predominant breastfeeding rates}

In Table 2, we report the quality assessment of breastfeeding promotion intervention on predominant breast 
Table 1 Summary of findings for the effect of breastfeeding promotion interventions on exclusive breastfeeding rates.

\begin{tabular}{|c|c|c|c|c|c|c|c|c|c|}
\hline \multicolumn{6}{|c|}{ Quality Assessment } & \multicolumn{4}{|c|}{ Summary of Findings } \\
\hline & \multicolumn{2}{|l|}{ Directness } & \multicolumn{7}{|l|}{ No of events } \\
\hline $\begin{array}{l}\text { No of } \\
\text { Studies }\end{array}$ & Design & Limitations & Consistency & $\begin{array}{l}\text { Generalizability } \\
\text { to population } \\
\text { of interest }\end{array}$ & $\begin{array}{l}\text { Generalizability } \\
\text { to intervention } \\
\text { of interest }\end{array}$ & Intervention & Control & $\begin{array}{l}\text { Relative } \\
\text { Risk } \\
(95 \% \mathrm{Cl})\end{array}$ & Comments \\
\hline \multicolumn{10}{|c|}{ Rate of exclusive breastfeeding at day 1: low outcome-specific quality } \\
\hline 15 & $\begin{array}{l}6 \text { RCTs }[14,40,49,70,71,74], 9 \text { QE } \\
{[21,32,45,48,56,59,64,85,88]}\end{array}$ & $\begin{array}{l}\text { Studies used different follow } \\
\text { up periods and recall criteria. } \\
\text { Mothers in the intervention } \\
\text { group may have over- } \\
\text { reported feeding practices. }\end{array}$ & $\begin{array}{l}6 \text { of } 15 \\
\text { studies } \\
\text { suggest } \\
\text { benefit. } \\
\text { Significant } \\
\text { heterogeneity }\end{array}$ & $\begin{array}{l}10 \text { out of } 15 \\
\text { studies were } \\
\text { conducted in } \\
\text { developed } \\
\text { countries }\end{array}$ & $\begin{array}{l}\text { Pooled results } \\
\text { for different } \\
\text { types of } \\
\text { interventions. }\end{array}$ & 4093 & 6316 & $\begin{array}{l}1.43 \\
{[1.09} \\
1.87]\end{array}$ & $\begin{array}{l}\text { Random effects } \\
\text { meta-analysis } \\
\text { due to } \\
\text { heterogeneity. } \\
\text { The majority of } \\
\text { studies used } \\
\text { individual } \\
\text { counseling as } \\
\text { the intervention; } \\
\text { most were } \\
\text { facility and } \\
\text { community- } \\
\text { based. }\end{array}$ \\
\hline \multicolumn{10}{|c|}{ Rate of exclusive breastfeeding at 0-1 month: Low outcome-specific quality } \\
\hline 30 & $\begin{array}{l}22 \text { RCTs, } 8 \text { QE } \\
{[13,17-19,21,22,33,38,39,41,42,45,52,53,55-57,60,61} \\
65,66,68,71-74,76,81,84,85]\end{array}$ & $\begin{array}{l}\text { Studies used different follow } \\
\text { up periods. Recall criteria } \\
\text { variable across studies (past } \\
24 \text { hr, past week or previous } \\
\text { month). Mothers in the } \\
\text { intervention group may have } \\
\text { over-reported feeding } \\
\text { practices. }\end{array}$ & $\begin{array}{l}15 \text { of } 30 \\
\text { studies } \\
\text { suggest } \\
\text { benefit. } \\
\text { Significant } \\
\text { heterogeneity }\end{array}$ & $\begin{array}{l}19 \text { of } 30 \text { studies } \\
\text { were conducted } \\
\text { in developed } \\
\text { countries }\end{array}$ & $\begin{array}{l}\text { Pooled results } \\
\text { for different } \\
\text { types of } \\
\text { interventions }\end{array}$ & 1512 & 1276 & $\begin{array}{l}1.30 \\
{[1.19} \\
1.42]\end{array}$ & $\begin{array}{l}\text { Random effects } \\
\text { meta-analysis } \\
\text { due to } \\
\text { heterogeneity. } \\
\text { The majority of } \\
\text { studies used } \\
\text { individual } \\
\text { counseling as } \\
\text { the intervention; } \\
\text { most were } \\
\text { facility and } \\
\text { community- } \\
\text { based. }\end{array}$ \\
\hline \multicolumn{10}{|c|}{ Rate of exclusive breastfeeding at 1-6 months: low outcome-specific quality } \\
\hline 53 & $\begin{array}{l}34 \text { RCTs, } 19 \text { QE [11-14,19,20,22,33-38, } \\
40-52,54,57-59,61-65,67,69,71-83,85-87]\end{array}$ & $\begin{array}{l}\text { Variable follow up periods } \\
\text { used in studies. Recall criteria } \\
\text { variable across studies (past } \\
24 \mathrm{hr} \text {, past week or previous } \\
\text { month). Mothers in the } \\
\text { intervention group may have } \\
\text { over-reported feeding } \\
\text { practices. }\end{array}$ & $\begin{array}{l}21 \text { of } 53 \\
\text { studies } \\
\text { suggest } \\
\text { benefit. } \\
\text { Significant } \\
\text { heterogeneity }\end{array}$ & $\begin{array}{l}29 \text { of } 53 \text { studies } \\
\text { were conducted } \\
\text { in developed } \\
\text { countries }\end{array}$ & $\begin{array}{l}\text { Pooled results } \\
\text { for different } \\
\text { types of } \\
\text { interventions }\end{array}$ & 5481 & 4897 & $\begin{array}{l}1.90 \\
{[1.54} \\
2.34]\end{array}$ & $\begin{array}{l}\text { Random effects } \\
\text { meta-analysis } \\
\text { due to } \\
\text { heterogeneity. } \\
\text { The majority of } \\
\text { studies used } \\
\text { individual } \\
\text { counseling as } \\
\text { the intervention; } \\
\text { most were } \\
\text { facility and } \\
\text { community- } \\
\text { based }\end{array}$ \\
\hline
\end{tabular}




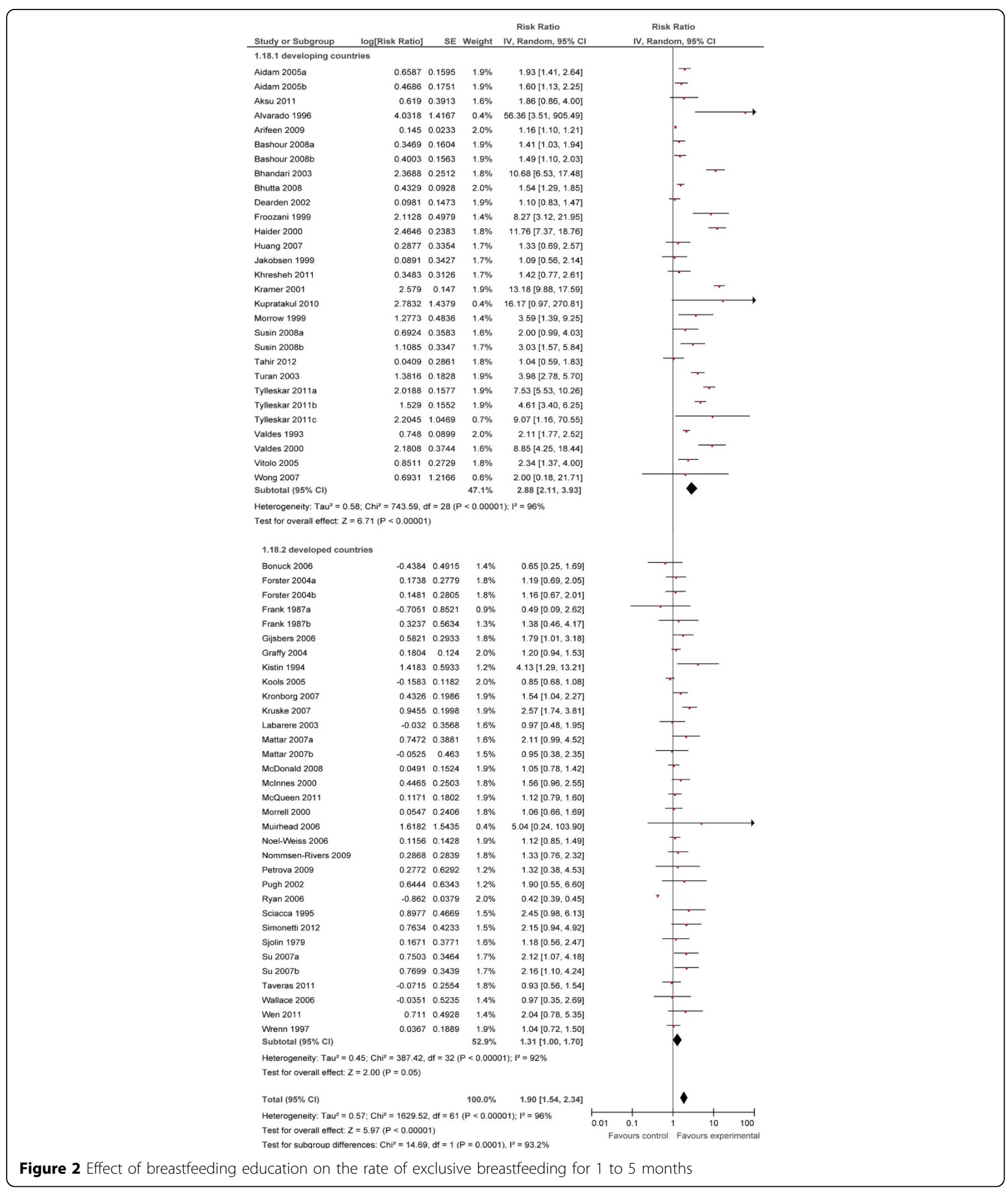

feeding. 13 studies had reported this outcome $[11,12,18$, $20,36,42,46,52,58,61,72,76,85]$ and eight of these were conducted in developing countries. Overall, educational interventions had a non-significant effect on predominant breastfeeding rates at $<1$ month (RR: $0.66,95 \% \mathrm{CI}$ :
$0.43,1.01)$ and at $1-5$ months (RR: 1.08 , 95\%CI: 0.55 , 2.13) (Figure 3), while there were no studies reporting predominant breastfeeding rates at day 1 . Subgroup analysis also did not show significant findings for any of the subgroups. 
Table 2 Summary of findings for the effect of breastfeeding promotion interventions on predominant and partial breastfeeding rates.

\begin{tabular}{|c|c|c|c|c|c|c|c|c|c|}
\hline \multicolumn{6}{|c|}{ Quality Assessment } & \multicolumn{4}{|c|}{ Summary of Findings } \\
\hline & \multicolumn{2}{|l|}{ Directness } & \multicolumn{7}{|l|}{ No of events } \\
\hline $\begin{array}{l}\text { No of } \\
\text { studies }\end{array}$ & Design & Limitations & Consistency & $\begin{array}{l}\text { Generalizability } \\
\text { to population } \\
\text { of interest }\end{array}$ & $\begin{array}{l}\text { Generalizability } \\
\text { to intervention } \\
\text { of interest }\end{array}$ & Intervention & Control & $\begin{array}{l}\text { Relative } \\
\text { Risk } \\
(95 \% \mathrm{Cl}) \\
\end{array}$ & Comments \\
\hline \multicolumn{10}{|c|}{ Rate of predominant breastfeeding at $<1$ month: Moderate outcome-specific quality } \\
\hline 6 & 5 RCTs, 1 QE $[18,52,61,72,76,85]$ & $\begin{array}{l}\text { Variable follow up periods } \\
\text { used in studies. Recall } \\
\text { criteria variable across } \\
\text { studies (past } 24 \text { hr, past } \\
\text { week or previous month). } \\
\text { mothers in the } \\
\text { intervention group may } \\
\text { have over-reported feeding } \\
\text { practices }\end{array}$ & $\begin{array}{l}\text { None of the } \\
\text { studies } \\
\text { suggest } \\
\text { benefit. } \\
\text { Insignificant } \\
\text { heterogeneity }\end{array}$ & $\begin{array}{l}3 \text { studies were } \\
\text { conducted in } \\
\text { developing } \\
\text { countries }\end{array}$ & $\begin{array}{l}\text { Pooled results } \\
\text { for different } \\
\text { types of } \\
\text { interventions }\end{array}$ & 33 & 59 & $\begin{array}{l}0.66 \\
{[0.43,} \\
1.01]\end{array}$ & $\begin{array}{l}\text { Fixed effects } \\
\text { meta-analysis; } \\
\text { insignificant } \\
\text { heterogeneity. } \\
\text { Most studies } \\
\text { were facility- } \\
\text { based and all } \\
\text { used } \\
\text { individual } \\
\text { counseling. }\end{array}$ \\
\hline \multicolumn{10}{|c|}{ Rate of predominant breastfeeding at 1- 5 months: low outcome specific-quality } \\
\hline 13 & 10 RCTs, 3 QE $[11,12,18,20,36,42,46,52,58,61,72,76,85]$ & $\begin{array}{l}\text { Variable follow up periods } \\
\text { used in studies. Recall } \\
\text { criteria variable across } \\
\text { studies (past } 24 \text { hr, past } \\
\text { week or previous month). } \\
\text { mothers in the } \\
\text { intervention group may } \\
\text { have over-reported feeding } \\
\text { practices }\end{array}$ & $\begin{array}{l}2 \text { studies } \\
\text { suggest } \\
\text { benefit. } \\
\text { Significant } \\
\text { heterogeneity }\end{array}$ & $\begin{array}{l}8 \text { of } 13 \text { studies } \\
\text { were conducted } \\
\text { in developed } \\
\text { countries }\end{array}$ & $\begin{array}{l}\text { Pooled results } \\
\text { for different } \\
\text { types of } \\
\text { interventions }\end{array}$ & 1433 & 707 & $\begin{array}{l}1.08 \\
{[0.55} \\
2.13]\end{array}$ & $\begin{array}{l}\text { Random } \\
\text { effects meta- } \\
\text { analysis due } \\
\text { to significant } \\
\text { heterogeneity. } \\
\text { Most studies } \\
\text { are facility- } \\
\text { based and } \\
\text { used } \\
\text { individual } \\
\text { counseling. }\end{array}$ \\
\hline \multicolumn{10}{|c|}{ Rate of partial breastfeeding at day 1: low outcome-specific quality } \\
\hline 6 & $2 \mathrm{RCT}, 4 \mathrm{QE}[45,49,59,64,71,89]$ & $\begin{array}{l}\text { Variable follow up periods } \\
\text { used in studies. Recall } \\
\text { criteria variable across } \\
\text { studies (past } 24 \text { hr, past } \\
\text { week or previous month). } \\
\text { Mothers in the intervention } \\
\text { group may have over- } \\
\text { reported feeding practices. }\end{array}$ & $\begin{array}{l}1 \text { of } 6 \text { studies } \\
\text { suggests } \\
\text { benefit. } \\
\text { Significant } \\
\text { heterogeneity }\end{array}$ & $\begin{array}{l}1 \text { of } 6 \text { studies } \\
\text { was conducted } \\
\text { in a developing } \\
\text { country }\end{array}$ & $\begin{array}{l}\text { Pooled results } \\
\text { for different } \\
\text { types of } \\
\text { interventions }\end{array}$ & 101 & 99 & $\begin{array}{l}1.21 \\
{[0.79} \\
1.87]\end{array}$ & $\begin{array}{l}\text { Random } \\
\text { effects meta- } \\
\text { analysis due } \\
\text { to significant } \\
\text { heterogeneity. } \\
\text { Most studies } \\
\text { used } \\
\text { individual } \\
\text { counseling } \\
\text { and most } \\
\text { were facility- } \\
\text { based. }\end{array}$ \\
\hline
\end{tabular}




\section{Table 2 Summary of findings for the effect of breastfeeding promotion interventions on predominant and partial breastfeeding rates. (Continued)}

\begin{tabular}{|c|c|c|c|c|c|c|c|c|c|}
\hline 11 & 8 RCTs, 3 QE $[18,19,45,52,61,66,71,72,76,85,88]$ & $\begin{array}{l}\text { Variable follow up periods } \\
\text { used in studies. Recall } \\
\text { criteria variable across } \\
\text { studies (past } 24 \mathrm{hr} \text {, past } \\
\text { week or previous month). } \\
\text { mothers in the } \\
\text { intervention group may } \\
\text { have over-reported feeding } \\
\text { practices. }\end{array}$ & $\begin{array}{l}\text { None of the } \\
\text { studies } \\
\text { suggest } \\
\text { benefit. } \\
\text { Insignificant } \\
\text { heterogeneity }\end{array}$ & $\begin{array}{l}5 \text { of } 11 \text { studies } \\
\text { were conducted } \\
\text { in developing } \\
\text { countries }\end{array}$ & $\begin{array}{l}\text { Pooled results } \\
\text { for different } \\
\text { types of } \\
\text { interventions }\end{array}$ & 112 & 151 & $\begin{array}{l}0.88 \\
{[0.72,} \\
1.08]\end{array}$ & $\begin{array}{l}\text { Fixed effects } \\
\text { meta-analysis; } \\
\text { insignificant } \\
\text { heterogeneity } \\
\text { Most studies } \\
\text { used } \\
\text { individual } \\
\text { counseling } \\
\text { and most } \\
\text { were facility- } \\
\text { based. }\end{array}$ \\
\hline \multicolumn{10}{|c|}{ Rate of partial breastfeeding at 1-5 months: moderate outcome-specific quality } \\
\hline 20 & $\begin{array}{l}11 \text { RCTs, } 9 \text { QE } \\
{[18-20,36,42,45,47,49,51,52,59,61,62,71,72,76,80,85,86,89]}\end{array}$ & $\begin{array}{l}\text { Variable follow up periods } \\
\text { used in studies. Recall } \\
\text { criteria variable across } \\
\text { studies (past } 24 \text { hr, past } \\
\text { week or previous month). } \\
\text { mothers in the } \\
\text { intervention group may } \\
\text { have over-reported feeding } \\
\text { practices. }\end{array}$ & $\begin{array}{l}\text { None of the } \\
\text { studies } \\
\text { suggest } \\
\text { benefit. } \\
\text { Significant } \\
\text { heterogeneity }\end{array}$ & $\begin{array}{l}9 \text { of } 20 \text { studies } \\
\text { were conducted } \\
\text { in developing } \\
\text { countries }\end{array}$ & $\begin{array}{l}\text { Pooled results } \\
\text { for different } \\
\text { types of } \\
\text { interventions }\end{array}$ & 524 & 578 & $\begin{array}{l}0.87 \\
{[0.75,} \\
1.02]\end{array}$ & $\begin{array}{l}\text { Random } \\
\text { effects meta- } \\
\text { analysis due } \\
\text { to significant } \\
\text { heterogeneity. } \\
\text { Most studies } \\
\text { used } \\
\text { individual } \\
\text { counseling } \\
\text { and most } \\
\text { were facility- } \\
\text { based. }\end{array}$ \\
\hline
\end{tabular}




\section{Partial breastfeeding rates}

In Table 2, we report the quality assessment of breastfeeding promotion intervention on partial breast feeding. 24 studies $[18-20,34,36,42,45,47,49,51,52,59,61,62,64$, $66,71,72,76,80,85,86,88,89]$ reported outcomes of partial breastfeeding, of which ten were conducted in developing countries. Overall, educational interventions had a non-significant effect on partial breastfeeding rates at day 1 (RR: $1.2195 \%$ CI: $0.79,1.87$ ), at <1 month (RR: 0.88 95\% CI: $0.72,1.08$ ) and at $1-5$ months (RR: 0.87, 95\%CI: $0.75,1.02$ ) intervals (Figure 4 ).

For the subgroup analysis based on the level of care, combined facility and community-based interventions had a significant reduction of $66 \%$ (RR: $0.34,95 \% \mathrm{CI}$ : $0.13-0.93)$ at $<1$ month duration. Findings from all other subgroups were non-significant.

\section{'No breastfeeding' rates}

In Table 3, we report the quality assessment of breastfeeding promotion intervention on 'no breast feeding'. Of the 97 papers [10-14,17-20,33,35-38,40-42,44,45, $47,49,51-55,57-74,76-84,86,88-130]$ reporting this particular outcome, 23 were from developing countries. Overall, educational interventions significantly decreased rates of no breastfeeding by $32 \%$ at day 1 (RR: $0.68,95 \%$ CI: $0.54-0.87$ ), 30\% (RR: $0.70,95 \%$ CI: $0.62-0.80$ ) at $<1$ month and $18 \%$ (RR: $0.82,95 \% \mathrm{CI}: 0.77-0.89$ ) at $1-5$ months intervals (Figure 5).

At day 1, subgroup analyses for type of counseling revealed that group counseling alone resulted in a $43 \%$ reduction (RR: 0.57, 95\% CI: 0.41-0.80) and individual counseling alone led to a $27 \%$ reduction (RR: $0.73,95 \%$ CI: 0.55-0.96). The effect of combined individual and 
group counseling was non-significant. Only facilitybased interventions led to a significant reduction of 52\% (RR: 0.48, 95\% CI: 0.34-0.69); the effects of communitybased and combined facility- and community-based interventions were non-significant. Interventions in both developing and developed countries had significant results, with reduction of $42 \%$ (RR: 0.58 , 95\% CI: $0.44-$ 0.78 ) and $27 \%$ (RR: 0.73, 95\% CI: 0.57-0.95), respectively.

At $<1$ month, subgroup analyses for type of counseling showed that combined individual and group counseling resulted in a $34 \%$ decrease (RR: $0.66,95 \%$ CI: 0.51-0.87), individual counseling alone resulted in a $29 \%$ decrease (RR: 0.71, 95\% CI: 0.61-0.84) and group counseling alone led to a $29 \%$ decrease (RR: 0.71 , 95\% CI: $0.51-$ 0.99). In the subgroup analyses for level of care, the effects of facility-based interventions and combined facility- and community-based interventions were $32 \%$ (RR: 0.68, 95\% CI: 0.56-0.83) and 33\% (RR: 0.67, 95\% CI: 0.54-0.83) respectively. The effects of communitybased interventions were non-significant. Developing 
Table 3 Summary of findings for effect of breastfeeding promotion interventions on 'no breastfeeding' rates.

\begin{tabular}{|c|c|c|c|c|c|c|c|c|c|}
\hline \multicolumn{3}{|c|}{ Quality Assessment } & & & & \multicolumn{4}{|c|}{ Summary of Findings } \\
\hline \multirow[b]{2}{*}{$\begin{array}{l}\text { No of } \\
\text { Studies }\end{array}$} & \multicolumn{2}{|l|}{ Directness } & \multicolumn{7}{|l|}{ No of events } \\
\hline & Design & Limitations & Consistency & $\begin{array}{l}\text { Generalizability } \\
\text { to population } \\
\text { of interest }\end{array}$ & $\begin{array}{l}\text { Generalizability } \\
\text { to intervention } \\
\text { of interest }\end{array}$ & Intervention & Control & $\begin{array}{l}\text { Relative } \\
\text { Risk } \\
(95 \% \mathrm{Cl})\end{array}$ & Comments \\
\hline \multicolumn{10}{|c|}{ Rate of no breastfeeding at day 1: low outcome-specific quality } \\
\hline 38 & $\begin{array}{l}21 \text { RCTs, } 17 \text { QE }[11,13,14,17,33,36,37,44,49,59,63,64, \\
68-71,74,78,79,89-91,96,100,103,107,108,110,111 \\
113,117,120-122,124-127]\end{array}$ & $\begin{array}{l}\text { Variable follow up } \\
\text { periods used in } \\
\text { studies. Recall criteria } \\
\text { variable across studies } \\
\text { (past } 24 \text { hr, past week } \\
\text { or previous month). }\end{array}$ & $\begin{array}{l}\text { Most studies } \\
\text { suggest } \\
\text { benefit. } \\
\text { Significant } \\
\text { heterogeneity }\end{array}$ & $\begin{array}{l}10 \text { of } 38 \text { studies } \\
\text { were conducted } \\
\text { in developing } \\
\text { countries }\end{array}$ & $\begin{array}{l}\text { Pooled results } \\
\text { for different } \\
\text { types of } \\
\text { interventions }\end{array}$ & 48026 & 39843 & $\begin{array}{l}0.68 \\
{[0.54} \\
0.87]\end{array}$ & $\begin{array}{l}\text { Random effects } \\
\text { meta-analysis } \\
\text { due to } \\
\text { significant } \\
\text { heterogeneity } \\
\text { Most studies } \\
\text { used individual } \\
\text { counseling and } \\
\text { most were } \\
\text { facility and } \\
\text { community- } \\
\text { based. } \\
\text { Effect of benefit } \\
\text { refers to } \\
\text { decrease in } \\
\text { numbers not } \\
\text { breastfeeding. }\end{array}$ \\
\hline \multicolumn{10}{|c|}{ Rate of no breastfeeding at $<1$ month: low outcome-specific quality } \\
\hline 33 & $\begin{array}{l}21 \text { RCTs, } 12 \text { QE }[10,18,19,38,45,52,53,61,63,66,68,71-74,76, \\
88,90,91,93,95,98,99,101-103,106,113,117,118,120,124,128]\end{array}$ & $\begin{array}{l}\text { Variable follow up } \\
\text { periods used in } \\
\text { studies. Recall criteria } \\
\text { variable across studies } \\
\text { (past } 24 \text { hr, past week } \\
\text { or previous month). }\end{array}$ & $\begin{array}{l}10 \text { of } 33 \\
\text { studies } \\
\text { suggest } \\
\text { benefit. } \\
\text { Significant } \\
\text { heterogeneity }\end{array}$ & $\begin{array}{l}4 \text { of } 33 \text { studies } \\
\text { were conducted } \\
\text { in developing } \\
\text { countries }\end{array}$ & $\begin{array}{l}\text { Pooled results } \\
\text { for different } \\
\text { types of } \\
\text { interventions }\end{array}$ & 770 & 1018 & $\begin{array}{l}0.70 \\
{[0.62} \\
0.80]\end{array}$ & $\begin{array}{l}\text { Random effects } \\
\text { meta-analysis } \\
\text { due to } \\
\text { significant } \\
\text { heterogeneity. } \\
\text { Most studies } \\
\text { used individual } \\
\text { counseling. } \\
\end{array}$ \\
\hline \multicolumn{10}{|c|}{ Rate of no breastfeeding at 1-5 months: low outcome-specific quality } \\
\hline 73 & $\begin{array}{l}41 \text { RCTs, } 32 \text { QE } \\
{[10,12,13,17-20,35,36,38,40-42,44,45,47,49} \\
51,52,54,58,59,61-64,67-69,71-74,76,78-83,86,90-93,95-99 \\
101,103-106,108-110,112-121,123,124,126,129,130]\end{array}$ & $\begin{array}{l}\text { Variable follow up } \\
\text { periods used in } \\
\text { studies. Recall criteria } \\
\text { variable across studies } \\
\text { (past } 24 \text { hr, past week } \\
\text { or previous month). }\end{array}$ & $\begin{array}{l}25 \text { of } 73 \\
\text { studies } \\
\text { suggest } \\
\text { benefit. } \\
\text { Significant } \\
\text { heterogeneity }\end{array}$ & $\begin{array}{l}16 \text { of } 73 \text { studies } \\
\text { were conducted } \\
\text { in developing } \\
\text { countries }\end{array}$ & $\begin{array}{l}\text { Pooled results } \\
\text { for different } \\
\text { types of } \\
\text { interventions }\end{array}$ & 15473 & 17578 & $\begin{array}{l}0.82 \\
{[0.77} \\
0.89]\end{array}$ & $\begin{array}{l}\text { Random effects } \\
\text { meta-analysis } \\
\text { due to } \\
\text { significant } \\
\text { heterogeneity } \\
\text { Most studies } \\
\text { used individual } \\
\text { counseling. }\end{array}$ \\
\hline
\end{tabular}




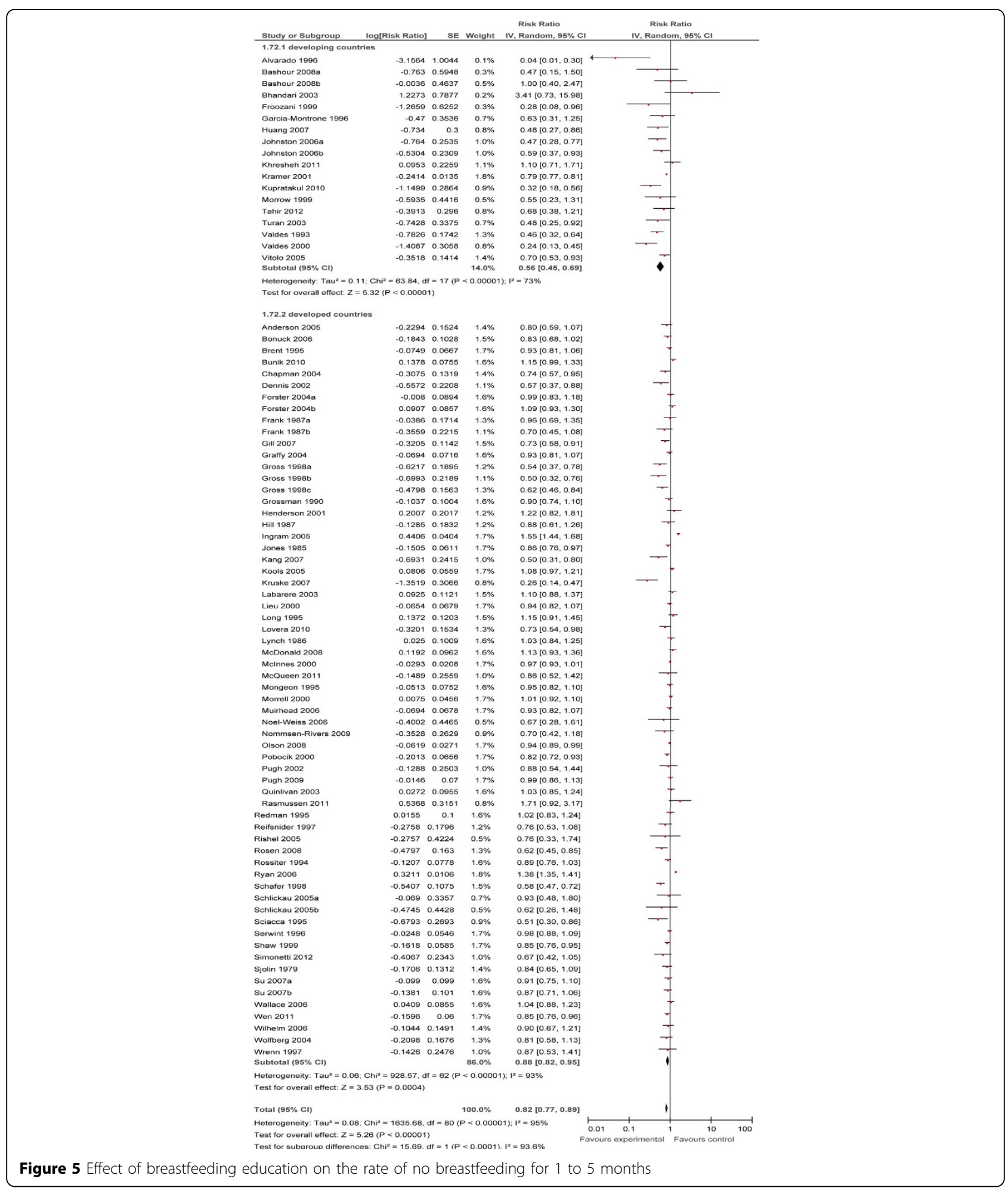

countries showed a reduction of $49 \%$ (RR: $0.51,95 \% \mathrm{CI}$ : $0.29-0.90)$ and in developed countries there was a reduction of 29\% (RR: 0.71, 95\% CI: 0.62-0.81).

For the 1-5 months interval, subgroup analyses showed statistically significant reduction in 'no breastfeeding' rates for combined individual and group counseling with a reduction of $32 \%$ (RR: $0.68,95 \%$ CI: $0.50-0.92$ ), individual counseling alone with a reduction of $14 \%$ (RR: $0.86,95 \%$ CI: 0.79-0.94), and group counseling alone with a reduction of 24\% (RR: 0.76, 95\% CI: 0.63-0.91). Facility-based 
and combined facility- and community-based interventions led to significant reduction of $18 \%$ (RR: 0.82 , 95\% CI: $0.75-0.89$ ) and $17 \%$ (RR: $0.83,95 \%$ CI: 0.75-0.93), respectively; however, results for community based interventions were non-significant. The effect of educational interventions in both developing and developed countries were significant at 44\% (RR: 0.56, 95\% CI: 0.45-0.69) and 12\% (RR: $0.88,95 \%$ CI: 0.82-0.95) respectively.

\section{Beyond 6 months}

Beyond 6 months, data was available from 11 studies $[12,34,38,81,83,89,94,98,104,108,113]$ for exclusive, partial and no breastfeeding rates. At 6-12 months, a 19\% increase in partial breastfeeding rates was demonstrated, which was significant (RR: 1.19, 95\% CI: 1.12-1.26). The effect of interventions was non-significant for both exclusive and no breastfeeding rates.

\section{Recommendations for LiST model}

Considering only the estimates from studies conducted in developing countries (Table 4), we propose that educational interventions increase EBF rates at day 1 by 157\% (RR: 2.57 95\% CI: 1.39, 4.77), at <1 month by $35 \%$ (RR: $1.35,95 \%$ CI: $1.15,1.58$ ) and at $1-5$ months by 188\% (RR: 2.88, 95\% CI: 2.11, 3.93). For predominant and partial breastfeeding rates, results were non-significant for all age durations. For 'no breastfeeding' we propose that educational interventions are associated with a reduction of $42 \%$ (RR: $0.58,95 \% \mathrm{CI}: 0.44,0.78$ ) at day 1 , $49 \%$ (RR: $0.51,95 \%$ CI: $0.29,0.90$ ) for $<1$ month and 44\% (RR: 0.56, 95\% CI: 0.45, 0.69) for 1-5 month ages.

\section{Discussion}

In this systematic review we summarized the effect of educational interventions to promote breastfeeding. We specifically examined the effect of these interventions on the various categories of breastfeeding, i.e. exclusive, predominant, partial breastfeeding and no breastfeeding, at day 1, $<1$ month, and 1-5 months. We also observed the prevalence of breastfeeding beyond 6 months if any study had reported outcomes in this age range.

EBF rates appeared to increase as a result of breastfeeding promotion interventions by $43 \%$ at day 1 , by $30 \%$ till 1 month, and by $90 \%$ from 1-5 months (low outcome-specific quality of evidence). Significant reduction in rates of no breastfeeding were observed for the same time intervals, i.e. by $32 \%$ at day 1 , by $30 \%$ till 1 month, and by $18 \%$ from 1-5 months (low outcome-specific quality of evidence). The overall effects of these interventions on predominant and partial breastfeeding rates were non-significant (moderate outcome-specific quality of evidence).

Combined individual and group counseling was found more effective than individual or group counseling alone. Overall, facility and combined facility- and community-based interventions led to greater improvements in breastfeeding rates, except for EBF at 1-5 months when the greatest increase resulted from communitybased interventions. The effects of interventions in developing countries were greater than those observed in developed countries, i.e. increases in EBF rates of 35\% compared to $26 \%$ at $<1$ month. At day 1 and at $1-5$ months, the effects of interventions in developing countries on EBF rates were increases of $157 \%$ and $188 \%$, respectively, whereas results for developed countries were non-significant. Reduction in 'no breastfeeding' rates of $42 \%$ were demonstrated in developing countries compared to $27 \%$ in developed countries at day 1, $49 \%$ compared to $29 \%$ at $<1$ month, and $44 \%$ compared to $12 \%$ at 1-5 months. Beyond 6 months, educational interventions had no significant effect except increasing rates of partial breastfeeding by $19 \%$ (moderate outcome-specific quality of evidence).

One of the limitations of this review is that the methodology of the RCTs included, indicated an unclear or high risk of bias as most RCTs demonstrated unclear

Table 4 Estimates of effect of breastfeeding promotion interventions on exclusive, predominant, partial and no breastfeeding rates in developing countries: Recommendations for LiST model.

\begin{tabular}{ll}
\hline Feeding practice and time interval & Relative Risk (95\% Cl) \\
\hline Exclusive breastfeeding rate at day 1 & $2.57[1.39,4.77]$ \\
Exclusive breastfeeding rate at $<1$ month & $1.35[1.15,1.58]$ \\
Exclusive breastfeeding rate at $1-5$ months & $2.88[2.11,3.93]$ \\
Predominant breastfeeding rate at $<1$ month & $0.67[0.42,1.06]$ \\
Predominant breastfeeding rate at $1-5$ months & $1.23[0.49,3.08]$ \\
Partial breastfeeding rate at day 1 & $0.84[0.61,1.15]$ \\
Partial breastfeeding rate at $<1$ month & $0.94[0.72,1.24]$ \\
Partial breastfeeding rate at 1-5 months & $0.83[0.65,1.06]$ \\
'No breastfeeding' rate at day 1 & $0.58[0.44,0.78]$ \\
'No breastfeeding' rate at $<1$ month & $0.51[0.29,0.90]$ \\
'No breastfeeding' rate at $1-5$ months & $0.56[0.45,0.69]$ \\
\hline
\end{tabular}


blinding and/or allocation concealment. As quasi-experimental trials were also included, most of which did not employ blinding, this limited the quality of the evidence. Not only was there methodological heterogeneity across studies based on study design, clinical heterogeneity was also observed due to variations in types of intervention and the duration of the intervention, target population (differences in income and education), outcome definitions ('fully' breastfeeding interpreted as EBF but possibly including predominant $\mathrm{BF}$ ) and different time intervals for follow-up. There were also differences in exposure to intervention, e.g. in the availability of a breastfeeding support telephone service, all the mothers in the intervention group did not choose to use the service. To investigate the subsequent statistical heterogeneity, we performed subgroup analyses to identify the cause. The random effects model was used to address this heterogeneity when it could not be explained. Criteria for recall of infant feeding practices for mothers were also variable, e.g. ranging from 'continuous EBF from birth' to 'EBF in last 24 hours'.

Other reviews on the subject include a Cochrane review on antenatal education for increasing breastfeeding duration, which examined specific types of breastfeeding education and compared multiple methods with a single method of education. Peer counseling, lactation consultation and formal BF education during pregnancy were found to increase BF duration. Though we have included interventions given both during the antenatal and postpartum periods, our findings are similar with respect to the effectiveness of individual and group counseling, or individual counseling alone. Our findings are also similar to the previous review [25], which concluded that educational interventions increased EBF rates at 4-6 weeks and at 6 months, and the review by Chapman et al [23], which specifically examined studies with peer counseling programs and found that in the majority of studies peer counselors improved rates of breastfeeding initiation, duration and exclusivity.

We observed a statistically significant increase in EBF rates as well as a reduction in no breastfeeding rates at all measured time intervals till 6 months of age as a result of promotional interventions for breastfeeding. This corresponds with the messages in many interventions, which promote EBF till the age of 6 months in compliance with WHO recommendations [1]. A general effect of reduction of predominant and partial breastfeeding rates was demonstrated at day $1,<1$ month and 1-5 months, however results were broadly non-significant. This finding may be explained by the rise of EBF as mothers realize the importance of not introducing formula or non-nutritional water-based foods early in the life of the infant. One exception to this pattern was the significant increase in partial breastfeeding rates of
$325 \%$ (RR 4.25, CI 1.43-12.61) in the community-based interventions subgroup at day 1 , comprising a single quasi-experimental study [59].

The impact was greater in developing countries when compared to developed countries. This could be because in less developed health systems, routine breastfeeding education in-hospital or follow-up home visits from public health nurses are less common than in the developed world leading to gaps in mothers' knowledge of breastfeeding. These mothers may benefit more after any educational intervention. Breastfeeding is also socially accepted as the norm in many cultures in developing countries, which would make mothers more eager to breastfeed after counseling. Mothers in developed countries may have increases in breastfeeding rates of lower magnitude due to wider availability of formula, work constraints and social perceptions.

Both individual and group counseling markedly increased the rates of exclusive breastfeeding, with combined individual and group counseling having the greatest effect from 1-5 months of age with a $101 \%$ increase. Combined individual and group counseling also led to a greater decrease in no breastfeeding rates, of $34 \%$ till 1 month and $32 \%$ for $1-5$ months, than individual or group counseling alone. Receiving the combination of one-on-one educational sessions with group sessions may be the ideal combination for women as a motivating strategy to continue breastfeeding.

Observing the success of educational strategies for promoting breastfeeding in developing countries, we should consider introducing these strategies on a large scale, utilizing both facility-based care and resources at the community level. These interventions should involve well-timed individual counseling along with group sessions for helping mothers achieve the goal of EBF till 6 months and continued BF till two years of life.

\section{Conclusion}

Breastfeeding education and/or support increased EBF rates and decreased no breastfeeding rates at birth, $<1$ month and 1-5 months. Combined individual and group counseling appeared to be superior to individual or group counseling alone. Interventions in developing countries had a greater impact than those in developed countries. These interventions have the potential to obtain optimum breast feeding practices and should be scaled up.

\section{Additional material}

Additional File 1: Detailed quality of evidence tables for the subgroups.

Additional File 2: Plots for meta analyses for day 1 and 0-1 month. 


\section{Competing interests}

We do not have any financial or non-financial competing interests for this review. The publications costs for this paper are from a grant from the Bill \& Melinda Gates Foundation to the US Fund for UNICEF (grant 43386 to "Promote evidence-based decision making in designing maternal, neonatal, and child health interventions in low- and middle-income countries").

\section{Authors' contributions}

Dr ZAB was responsible for designing the review and coordinating the review. SH, JKD, Al and RAS were responsible for: data collection, screening the search results, screening retrieved papers against inclusion criteria, appraising quality of papers, abstracting data from papers, entering data into RevMan, analysis and interpretation of data and writing the review. ZAB, RAS and JKD critically reviewed and modified the manuscript.

\section{Acknowledgment}

This work was supported in part by a grant from the Bill \& Melinda Gates Foundation.

\section{Declarations}

The publication costs for this supplement were funded by a grant from the Bill \& Melinda Gates Foundation to the US Fund for UNICEF (grant 43386 to "Promote evidence-based decision making in designing maternal, neonatal, and child health interventions in low- and middle-income countries"). The Supplement Editor is the principal investigator and lead in the development of the Lives Saved Tool (LiST), supported by grant 43386 . He declares that he has no competing interests.

This article has been published as part of BMC Public Health Volume 13 Supplement 3, 2013: The Lives Saved Tool in 2013: new capabilities and applications. The full contents of the supplement are available online at http://www.biomedcentral.com/bmcpublichealth/supplements/13/S3.

\section{Authors' details}

${ }^{1}$ Division of Women \& Child Health, The Aga Khan University, Karachi 74800, Pakistan. ${ }^{2}$ Global Child Health and Policy, Centre for Global Child Health, The Hospital for Sick Children, Toronto, ON, Canada.

Published: 17 September 2013

\section{References}

1. WHO: The optimal duration of exclusive breastfeeding: report of an expert consultation. Geneva, Switzerland: World Health Organization; 2001, Available from: http://www.who.int/nutrition/publications/infantfeeding/ WHO_NHD_01.09/en/index.html.

2. Dimond $\mathrm{HJ}$, Ashworth A: Infant feeding practices in Kenya, Mexico and Malaysia. The rarity of the exclusively breast-fed infant. Hum Nutr Appl Nutr 1987, 41(1):51-64, Epub 1987/02/01. PubMed PMID: 3558008.

3. Perez-Escamilla R: Breastfeeding in Africa and the Latin American and Caribbean region: the potential role of urbanization. J Trop Pediatr 1994, 40(3):137-43, Epub 1994/06/01. PubMed PMID: 8078111.

4. UNICEF: A Report Card on Nutrition: Number 4. 2006

5. Cai X, Wardlaw T, Brown DW: Global trends in exclusive breastfeeding. Int Breastfeed J 2012, 7:12

6. Countdown to 2015. Maternal, Newborn and Child Survival: The 2012 Report. WHO and UNICEF; 2012.

7. Lamberti LM, Fischer Walker CL, Noiman A, Victora C, Black RE: Breastfeeding and the risk for diarrhea morbidity and mortality. BMC Public Health 2011, 11(Suppl 3):S15, Epub 2011/04/29. doi: 10.1186/14712458-11-s3-s15. PubMed PMID: 21501432; PubMed Central PMCID: PMCPMC3231888.

8. Thulier D, Mercer J: Variables associated with breastfeeding duration. J Obstet Gynecol Neonatal Nurs 2009, 38:259-68.

9. Chezem J, Friesen C, Boettcher J: Breastfeeding knowledge, breastfeeding confidence, and infant feeding plans: Effects on actual feeding practices. J Obstet Gynecol Neonatal Nurs 2003, 32:40-7.

10. Bunik M, Shobe P, O'Connor M, Beaty B, Langendoerfer S, Crane L, et al: Are 2 Weeks of Daily Breastfeeding Support Insufficient to Overcome the Influences of Formula? Academic Pediatrics 2010, 10:21-8.

11. Dearden K, Altaye M, Maza I, Oliva M, Stone-Jimenez M, Burkhalter BR, et al: The impact of mother-to-mother support on optimal breast-feeding: a controlled community intervention trial in peri-urban Guatemala City, Guatemala. Rev Panam Salud Publica 2002, 12(3):193-201.

12. Kramer MS, Chalmers B, Hodnett ED, Sevkovskaya Z, Dzikovich I, Shapiro S, et al: Promotion of breastfeeding intervention trial (PROBIT): a randomized trial in the Republic of Belarus. JAMA 2001, 285(4):413-20.

13. Morrow AL, Guerrero ML, Shults J, Calva JJ, Lutter C, Bravo J, et al: Efficacy of home-based peer counselling to promote exclusive breastfeeding: a randomised controlled trial. Lancet 1999, 353:1226-31.

14. Haider R, Ashworth A, Kabir I, Huttly SR: Effect of community-based peer counsellors on exclusive breastfeeding practices in Dhaka, Bangladesh: a randomised controlled trial. Lancet 2000, 356:1643-47.

15. WHO, UNICEF: Integrated management of childhood illness: chartbook and training course. Geneva: World Health Organization; 1995.

16. Santos I, Victora C, Martines J, Gonçalves H, Gigante D, Valle N, et al: Nutrition counseling increases weight gain among Brazilian children. Journal of Nutrition 2001, 131(11):2866-73.

17. Anderson A, Damio G, Young S, Chapman D, Perez-Escamilla R: A randomized trial assessing the efficacy of peer counseling on exclusive breastfeeding in a predominantly Latina low-income community. Arch Pediatr Adolesc Med 2005, 159(9):836-41, Epub 2005/09/07. doi: 159/9/836 [pii] 10.1001/archpedi.159.9.836. PubMed PMID: 16143742.

18. Dennis CL, Hodnett E, Gallop R, Chalmers B: The effect of peer support on breast-feeding duration among primiparous women: a randomized controlled trial. CMAJ 2002, 166(1):21-8, Epub 2002/01/22. PubMed PMID: 11800243.

19. Alvarado R, Atalah E, Diaz S, Rivero S, Labbe M, Escudero Y: Evaluation of a breastfeeding-support programme with health promoters' participation. Food Nutr Bull 1996, 17(1):49-53.

20. Noel-Weiss J, Rupp A, Gragg B, Bassett V, Woodend K: Randomized Controlled Trial to Determine Effects of Prenatal Breastfeeding Workshop on Maternal Breastfeeding Self-Efficacy and Breastfeeding Duration. JOGNN 2006, 35:616-24.

21. Lin SS, Chien LY, Tai CJ, Lee CF: Effectiveness of a prenatal education programme on breastfeeding outcomes in Taiwan. J Clin Nurs 2008, 17(3):296-303, Epub 2007/10/13. doi: JCN1927 [pii] 10.1111/j.13652702.2006.01927.x. PubMed PMID: 17931376

22. Aidam B, Perez-Escamilla R, Lartey A: Lactation Counseling Increases Exclusive Breast-Feeding Rates in Ghana. The Journal of Nutrition 2005, 135:1691-5

23. Chapman DJ, Morel K, Anderson AK, Damio G, Perez-Escamilla R: Breastfeeding peer counseling: from efficacy through scale-up. I Hum Lact 2010, 26(3):314-26, Epub 2010/08/19. PubMed PMID: 20715336; PubMed Central PMCID: PMCPMC3115698.

24. Lumbiganon P, Martis R, Laopaiboon M, Festin M, Ho J, Hakimi M: Antenatal breastfeeding education for increasing breastfeeding duration. Cochrane Pregnancy and Childbirth Group 2012.

25. Imdad A, Yakoob MY, Bhutta ZA: Effect of breastfeeding promotion interventions on breastfeeding rates, with special focus on developing countries. BMC Public Health 2011, 11(Suppl 3):S24, Epub 2011/04/29. doi: 10.1186/1471-2458-11-s3-s24. PubMed PMID: 21501442; PubMed Central PMCID: PMCPmc3231898

26. Walker N, Fischer-Walker C, Bryce J, Bahl R, Cousens S: Standards for CHERG reviews of intervention effects on child survival. Int J Epidemiol 2010, 39(Suppl 1):i21-31, Epub 2010/04/02. doi: dyq036 [pii] 10.1093/ije/ dyq036. PubMed PMID: 20348122.

27. Atkins $D$, Best D, Briss PA, Eccles M, Falck-Ytter $Y$, Flottorp $S$, et al: Grading quality of evidence and strength of recommendations. BMJ 2004, 328(7454):1490, Epub 2004/06/19. doi: 10.1136/bmj.328.7454.1490 328/ 7454/1490 [pii]. PubMed PMID: 15205295.

28. RevMan: The Cochrane Colloboration. Review Manager (RevMan) 5 for Windows. Oxford, England; 2003.

29. WHO: Indicators for assessing breastfeeding practices. WHO Geneva, Switzerland: WHO Document WHO/CDD/SER; 1991:91:14.

30. WHO: Indicators for Assessing Infant and Young Child Feeding Practices: Conclusions of a Consensus Meeting Held 6-8 November 2007 in Washington DC, USA. World Health Organization (WHO); 2008.

31. World Bank list of economies. 2009, Available from: http://siteresources. worldbank.org/DATASTATISTICS/Resources/CLASS.XLS.

32. Abolyan LV: The breastfeeding support and promotion in Baby-Friendly Maternity Hospitals and Not-as-Yet Baby-Friendly Hospitals in Russia. 
Breastfeed Med 2006, 1(2):71-8, Epub 2007/07/31. doi: 10.1089/bfm.2006.1.71. PubMed PMID: 17661566.

33. Aksu H, Küçük $M$, Düzgün $G$ : The effect of postnatal breastfeeding education/support offered at home 3 days after delivery on breastfeeding duration and knowledge: a randomized trial. J Matern Fetal Neonatal Med 2011, 24(2):354-61.

34. Arifeen $S$, Hoque D, Akter T, Rahman M, Hoque M, Begum $K$, et al: Effect of the Integrated Management of Childhood Illness strategy on childhood mortality and nutrition in a rural area in Bangladesh: a cluster randomised trial. Lancet 2009, 374(9687):393-403.

35. Bashour H, Kharouf M, Abdulsalam A, El Asmar K, Tabbaa M, Cheikha S: Effect of postnatal home visits on maternal/infant outcomes in Syria: a randomized controlled trial. Public Health Nurs 2008, 25(2):115-25.

36. Bhandari N, Bahl R, Mazumdar S, Martines J, Black R, Bhan M: Infant Feeding Study Group. Effect of community-based promotion of exclusive breastfeeding on diarrhoeal illness and growth: a cluster randomised controlled trial. Lancet 2003, 361(9367):1418-23.

37. Bhutta Z, Memon Z, Soofi S, Salat M, Cousens S, Martines J: Implementing community-based perinatal care: results from a pilot study in rural Pakistan. Bull World Health Organ 2008, 86(6):452-9.

38. Bonuck K, Freeman K, Trombley M: Randomized Controlled Trial of a Prenatal and Postnatal Lactation Consultant Intervention on Infant Health Care Use. Arch Pediatr Adolesc Med 2006, 160:953-60.

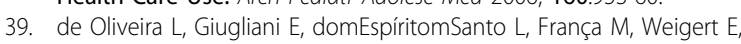

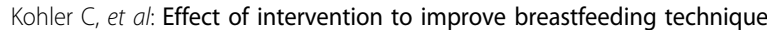
on the frequency of exclusive breastfeeding and lactation-related problems. J Hum Lact 2006, 22(3):315-21.

40. Forster D, McLachlan H, Lumley J, Beanland C, Waldenstrom U, Amir L: Two mid-pregnancy interventions to increase the initiation and duration of breastfeeding: a randomized controlled trial. Birth 2004, 31(3):176-82, Epub 2004/08/28. doi: 10.1111/j.0730-7659.2004.00302.x BIR302 [pii]. PubMed PMID: 15330879

41. Frank DA, Wirtz SJ, Sorenson JR, Heeren T: Commercial discharge packs and breast-feeding counseling: effects on infant-feeding practices in a randomized trial. Pediatrics 1987, 80(6):845-54, Epub 1987/12/01. PubMed PMID: 3684395.

42. Froozani MD, Permehzadeh K, Motlagh AR, Golestan B: Effect of breastfeeding education on the feeding pattern and health of infants in their first 4 months in the Islamic Republic of Iran. Bull World Health Organ 1999, 77(5):381-5, Epub 1999/06/11. PubMed PMID: 10361754

43. Gijsbers B, Mesters I, Knottnerus J, Kester A, Van Schayck C: The Success of an Educational Program to Promote Exclusive Breastfeeding for 6 Months in Families with a History of Asthma: A Randomized Controlled Trial. Pediatric Asthma, Allergy and Immunology 2006, 19(4):214-22.

44. Graffy J, Taylor J, Williams A, Eldridge S: Randomised controlled trial of support from volunteer counsellors for mothers considering breast feeding. BMJ 2004, 328(7430):26, Epub 2004/01/03. doi: 10.1136/ bmj.328.7430.26 328/7430/26 [pii]. PubMed PMID: 14703543

45. Huang M, Kuo S, Avery M, Chen W, Lin K, Gau M: Evaluating effects of a prenatal web-based breastfeeding education programme in Taiwan. J Clin Nurs 2007, 16(8):1571-9.

46. Jakobsen MS, Sodemann M, Mølbak K, Alvarenga I, Aaby P: Promoting breastfeeding through health education at the time of immunizations: a randomized trial from Guinea Bissau. P Acta Paediatr 1999, 88(7):741-7.

47. Khresheh R, Suhaimat A, Jalamdeh F, Barclay L: The effect of a postnatal education and support program on breastfeeding among primiparous women: a randomized controlled trial. Int J Nurs Stud 2011, 48(9):1058-65.

48. Kistin N, Abramson R, Dublin P: Effect of peer counselors on breastfeeding initiation, exclusivity, and duration among low-income urban women. J Hum Lact 1994, 10(1):11-5

49. Kools $E$, Thijs $C$, Kester A, van den Brandt $P$, de Vries $H$ : A breast-feeding promotion and support program a randomized trial in the Netherlands. Preventive Medicine 2005, 40(1):60-70.

50. Kronborg H, Vaeth M, Olsen J, Iversen L, Harder I: Effect of early postnatal breastfeeding support: a cluster-randomized community based trial. Acto Paediatr 2007, 96(7):1064-70, Epub 2007/05/26. doi: APA341 [pii] 10.1111/ j.1651-2227.2007.00341.x. PubMed PMID: 17524018.

51. Kruske S, Schmied V, Cook M: The 'Earlybird' gets the breastmilk: findings from an evaluation of combined professional and peer support groups to improve breastfeeding duration in the first eight weeks after birth. Matern Child Nutr 2007, 3(2):108-19.
52. Kupratakul J, Taneepanichskul S, Voramongkol N, Phupong V: A randomized controlled trial of knowledge sharing practice with empowerment strategies in pregnant women to improve exclusive breastfeeding during the first six months postpartum. J Med Assoc Thai 2010, 93(9):1009-18, Epub 2010/09/29. PubMed PMID: 20873071

53. Labarere J, Gelbert-Baudino N, Ayral AS, Duc C, Berchotteau M, Bouchon N, et al: Efficacy of breastfeeding support provided by trained clinicians during an early, routine, preventive visit: a prospective, randomized, open trial of 226 mother-infant pairs. Pediatrics 2005, 115(2):e139-46, Epub 2005/02/03. doi: 115/2/e139 [pii] 10.1542/peds.2004-1362. PubMed PMID: 15687421

54. Labarere J, Bellin V, Fourny M, Gagnaire J, Francois P, Pons J: Assessment of a structured in-hospital educational intervention addressing breastfeeding: a prospective randomised open trial. BJOG 2003, 110(9):847-52.

55. Langer A: Intrapartum Social Support and Exclusive Breastfeeding in Mexico. Wellstart International, Expanded Promotion of Breastfeeding Program Exclusive Breastfeeding Promotion: A Summary of Findings from EPB's Applied Research Program (1992-1996). 1996.

56. Lin CH, Kuo SC, Lin KC, Chang TY: Evaluating effects of a prenatal breastfeeding education programme on women with caesarean delivery in Taiwan. J Clin Nurs 2008, 17(21):2838-45, Epub 2008/07/16. doi: JCN2289 [pii] 10.1111/j.1365-2702.2008.02289.x. PubMed PMID: 18624780

57. Mattar CN, Chong YS, Chan YS, Chew A, Tan P, Chan YH, et al: Simple antenatal preparation to improve breastfeeding practice: a randomized controlled trial. Obstet Gynecol 2007, 109(1):73-80, Epub 2007/01/02. doi: 109/1/73 [pii] 10.1097/01.AOG.0000249613.15466.26. PubMed PMID: 17197590

58. McDonald SJ, Henderson JJ, Faulkner S, Evans SF, Hagan R: Effect of an extended midwifery postnatal support programme on the duration of breast feeding: A randomised controlled trial. Midwifery 2010, 26:88-100.

59. Mclnnes RJ, Love JG, Stone DH: Evaluation of a community-based intervention to increase breastfeeding prevalence. J Public Health Med 2000, 22(2):138-45, Epub 2000/07/27. PubMed PMID: 10912550

60. McKeever P, Stevens B, Miller K, MacDonell J, Gibbins S, Querriere D, et al: Home versus Hospital Breastfeeding Support for Newborns: A Randomized Controlled Trial. BIRTH 2002, 29(4):258-65.

61. McQueen K, Dennis C, Stremker R, Norman C: A Pilot Randomized Controlled Trial of a Breastfeeding Self-Efficacy Intervention With Primiparous Mothers. JOGNN 2011, 40:35-46.

62. Morrell CJ, Spiby H, Stewart P, Walters S, Morgan A: Costs and effectiveness of community postnatal support workers: randomised controlled trial. BMJ 2000, 321(7261):593-8, Epub 2000/09/08. PubMed PMID: 10977833.

63. Muirhead PE, Butcher G, Rankin J, Munley A: The effect of a programme of organised and supervised peer support on the initiation and duration of breastfeeding: a randomised trial. Br J Gen Pract 2006, 56(524):191-7, Epub 2006/03/16. PubMed PMID: 16536959

64. Nommsen-Rivers LA, Mastergeorge AM, Hansen RL, Cullum AS, Dewey KG: Doula care, early breastfeeding outcomes, and breastfeeding status at 6 weeks postpartum among low-income primiparae. J Obstet Gynecol Neonatal Nurs 2009, 38(2):157-73, Epub 2009/03/28. doi: JOGN1005 [pii] 10.1111/j.1552-6909.2009.01005.x. PubMed PMID: 19323712.

65. Petrova A, Ayers C, Stechna S, Gerling JA, Mehta R: Effectiveness of exclusive breastfeeding promotion in low-income mothers: a randomized controlled study. Breastfeed Med 2009, 4(2):63-9, Epub 2009/ 02/26. doi: 10.1089/bfm.2008.0126. PubMed PMID: 19239405.

66. Porteous R, Kaufman K, Rush J: The effect of individualized professional support on duration of breastfeeding: a randomized controlled trial. J Hum Lact 2000, 16(4):303-8, Epub 2001/01/13. PubMed PMID: 11155608.

67. Pugh LC, Milligan RA, Frick KD, Spatz D, Bronner Y: Breastfeeding duration, costs, and benefits of a support program for low-income breastfeeding women. Birth 2002, 29(2):95-100, Epub 2002/05/10. doi: 169 [pii]. PubMed PMID: 12000411

68. Rasmussen K, Dieterich C, Zelek S, Altabet J, Kjolhede C: Interventions to Increase the Duration of Breastfeeding in Obese Mothers: The Bassett Improving Breastfeeding Study. Breastfeeding Medicine 2011, 6(2):69-75.

69. Ryan A, Zhou W: Lower breastfeeding rates persist among the Special Supplemental Nutrition Program for Women, Infants, and Children participants, 1978-2003. Pediatrics 2006, 117(4):1136-46. 
70. Sandy J, Anisfeld E, Ramirez E: Effects of a prenatal intervention on breastfeeding initiation rates in a Latina immigrant sample. J Hum Lact 2009, 25(4):404-11.

71. Sciacca JP, Phipps BL, Dube DA, Ratliff Ml: Influences on breast-feeding by lower-income women: an incentive-based, partner-supported educational program. J Am Diet Assoc 1995, 95(3):323-8, Epub 1995/03/01. doi: S0002-8223(95)00083-6 [pii] 10.1016/S0002-8223(95)00083-6. PubMed PMID: 7860944.

72. Simonetti V, Palma E, Giglio A, Mohn A, Cicolini G: A structured telephonic counselling to promote the exclusive breastfeeding of healthy babies aged zero to six months: a pilot study. Int J Nurs Pract 2012, 18(3):289-94.

73. Sjolin S, Hofvander $Y$, Hillervik C: A prospective study of individual courses of breast feeding. Acta Paediatr Scand 1979, 68(4):521-9, Epub 1979/07/01. PubMed PMID: 463534.

74. Su LL, Chong YS, Chan YH, Chan YS, Fok D, Tun KT, et al: Antenatal education and postnatal support strategies for improving rates of exclusive breast feeding: randomised controlled trial. BMJ 2007, 335(7620):596, Epub 2007/08/03. doi: bmj.39279.656343.55 [pii] 10.1136/ bmj.39279.656343.55. PubMed PMID: 17670909.

75. Susin L, Giugliani E: Inclusion of fathers in an intervention to promote breastfeeding: impact on breastfeeding rates. J Hum Lact 2008, 24(4):386-92.

76. Tahir NM, Al-Sadat N: Does telephone lactation counselling improve breastfeeding practices?: A randomised controlled trial. Int J Nurs Stud 2013, 50:16-25.

77. Taveras E, Blackburn K, Gillman M, Haines J, McDonald J, Price S, et al: First steps for mommy and me: a pilot intervention to improve nutrition and physical activity behaviors of postpartum mothers and their infants. Matern Child Health J 2011, 15(8):1217-27.

78. Turan J, Say L: Community-based antenatal education in Istanbul, Turkey: effects on health behaviours. Health Policy Plan 2003, 18(4):391-8.

79. Valdes V, Perez A, Labbok M, Pugin E, Zambrano I, Catalan S: The Impact of a Hospital and Clinic-based Breastfeeding Promotion Programme in a Middle Class Urban Environment. Journal of Tropical Pediatrics 1993, 39:142-51.

80. Valdés V, Pugin E, Schooley J, Catalán S, Aravena R: Clinical support can make the difference in exclusive breastfeeding success among working women. J Trop Pediatr 2000, 46(3):149-54.

81. Vitolo M, Bortolini G, Feldens C, Drachle Mde L: [Impacts of the 10 Steps to Healthy Feeding in Infants: a randomized field trial]. Cad Saude Publica 2005, 21(5):1448-57.

82. Wallace LM, Dunn OM, Alder EM, Inch S, Hills RK, Law SM: A randomisedcontrolled trial in England of a postnatal midwifery intervention on breast-feeding duration. Midwifery 2006, 22(3):262-73, Epub 2005/12/29. doi: S0266-6138(05)00064-1 [pii] 10.1016/j.midw.2005.06.004. PubMed PMID: 16380197.

83. Wen L, Baur L, Simpson J, Rissel C, Flood V: Effectiveness of an early intervention on infant feeding practices and "tummy time": a randomized controlled trial. Arch Pediatr Adolesc Med 2011, 165(8):701-7.

84. Wiles LS: The effect of prenatal breastfeeding education on breastfeeding success and maternal perception of the infant. JOGN Nurs 1984, 13(4):253-7, Epub 1984/07/01. PubMed PMID: 6565110.

85. Wong $\mathrm{EH}$, Nelson E, Choi KC, Wong KP, Ip C, Ho LC: Evaluation of a peer counselling programme to sustain breastfeeding practice in Hong Kong. Int Breastfeed J 2007, 2:12, Epub 2007/09/22. doi: 1746-4358-2-12 [pii] 10.1186/1746-4358-2-12. PubMed PMID: 17883851.

86. Wrenn SE: Effects of a model-based intervention on breastfeeding attrition [dissertation]. San Antonio: University of Texas; 1997.

87. Tylleskär T, Jackson D, Meda N, Engebretsen IM, Chopra M, Diallo AH, et al: Exclusive breastfeeding promotion by peer counsellors in sub-Saharan Africa (PROMISE-EBF): a cluster-randomised trial. Lancet 2011, 378(9789):420-7.

88. Ryser F: Breastfeeding attitudes, intention, and initiation in low-income women: the effect of the best start program. Journal of Human Lactation 2004, 20(3):300-5.

89. Pisacane A, Continisio Gl, Aldinucci M, D'Amora S, Continisio P: A controlled trial of the father's role in breastfeeding promotion. Pediatrics 2005, 116(4):e494-e498.

90. Brent NB, Redd B, Dworetz A, D'Amico F, Greenberg JJ: Breast-feeding in a low-income population. Program to increase incidence and duration.
Arch Pediatr Adolesc Med 1995, 149(7):798-803, Epub 1995/07/01. PubMed PMID: 7795772.

91. Chapman DJ, Damio G, Young S, Perez-Escamilla R: Effectiveness of breastfeeding peer counseling in a low-income, predominantly Latina population: a randomized controlled trial. Arch Pediatr Adolesc Med 2004, 158(9):897-902, Epub 2004/09/08. doi: 10.1001/archpedi.158.9.897 158/9/897 [pii]. PubMed PMID: 15351756.

92. Garcia-Montrone $V$, Rose J: [An education experience for promoting breast-feeding and infant stimulation by low-income women: a preliminary study]. Cad Saude Publica 1996, 12(1):61-8.

93. Gill SL, Reifsnider E, Lucke JF: Effects of support on the initiation and duration of breastfeeding. West J Nurs Res 2007, 29(6):708-23, Epub 2007/ 06/15. doi: 0193945906297376 [pii] 10.1177/0193945906297376. PubMed PMID: 17557933.

94. Greiner T, Mitra SN: Evaluation of the effect of a breastfeeding message integrated into a larger communication project. J Trop Pediatr 1999, 45:351-7.

95. Gross S, Caulfield L, Bentley M, Bronner Y, Kessler L, Jensen J, et al: Counseling and motivational videotapes increase duration of breastfeeding in African-American WIC participants who initiate breastfeeding. J Am Diet Assoc 1998, 98:143-8.

96. Ingram J, Rosser J, Jackson D: Breastfeeding peer supporters and a community support group: evaluating their effectiveness. Matern Child Nutr 2005, 1:111-8

97. Johnston B, Huebner C, Anderson M, Tyll L, Thompson R: Healthy steps in an integrated delivery system: child and parent outcomes at 30 months. Arch Pediatr Adolesc Med 2006, 160(8):793-800.

98. Jones DA, West RR: Lactation nurse increases duration of breast feeding. Arch Dis Child 1985, 60(8):772-4, Epub 1985/08/01. PubMed PMID: 4037866.

99. Kang J, Choi S, Ryu E: Effects of a breastfeeding empowerment programme on Korean breastfeeding mothers: a quasi-experimental study. Int J Nurs Stud 2008, 45(1):14-23.

100. Kumar V, Mohanty S, Kumar A, Misra RP, Santosham M, Awasthi S, et al: Effect of community-based behaviour change management on neonatal mortality in Shivgarh, Uttar Pradesh, India: a cluster-randomised controlled trial. Lancet 2008, 372(9644):1151-62.

101. Lieu T, Braveman P, Escobar G, Fischer A, Jensvold N, Capra A: A Randomized Comparison of Home and Clinic Follow-Up Visits After Early Postpartum Hospital Discharge. Pediatrics 2000, 105(5):1058-65.

102. Lieu T, Wikler C, Capra A, Martin K, Escobar G, Braveman P: Clinical Outcomes and Maternal Perceptions of an Updated Model of Perinatal Care. Pediatrics 1998, 102(6):1437-44.

103. Long D, Funk-Archeluta M, Geiger C, Mozar A, Heins J: Peer Counselor Program Increases Breastfeeding Rates in Utah Native American WIC Population. Journal of Human Lactation 1995, 11(4):279-84.

104. Lovera D, Sanderson M, Bogle M, Vela Acosta M: Evaluation of a breastfeeding peer support program for fathers of Hispanic participants in a Texas special supplemental nutrition program for women, infants, and children. J Am Diet Assoc 2010, 110(11):1696-702.

105. Lynch SA, Koch AM, Hislop TG, Coldman AJ: Evaluating the effect of a breastfeeding consultant on the duration of breastfeeding. Can J Public Health 1986, 77(3):190-5, Epub 1986/05/01. PubMed PMID: 3742402.

106. Mongeon M, Allard R: [Controlled study of a regular telephone support program given by volunteers on the establishment of breastfeeding]. Can J Public Health 1995, 86(2):124-7, Epub 1995/03/01. PubMed PMID: 7757891.

107. Mottl-Santiago J, Walker C, Ewan J, Vragovic O, Winder S, Stubblefield P: A Hospital-Based Doula Program and Childbirth Outcomes in an Urban, Multicultural Setting. Matern Child Health J 2008, 12:372-7.

108. Olson BH, Haider SJ, Vangjel L, Bolton TA, Gold JG: A quasi-experimental evaluation of a breastfeeding support program for low income women in Michigan. Matern Child Health J 2010, 14(1):86-93, Epub 2008/12/17. doi: 10.1007/s10995-008-0430-5. PubMed PMID: 19082697.

109. Hill P: Effects of Education on Breastfeeding Success. Maternal-Child Nursing Journal 1987, 16(2):145-56.

110. Rishel P: Comparison of Breastfeeding Rates among Women Delivering Infants in Military Treatment Facilities with and without Lactation Consultants. Military Medicine 2005, 170(5):435-8.

111. Martens PJ: Does breastfeeding education affect nursing staff beliefs, exclusive breastfeeding rates, and Baby-Friendly Hospital Initiative 
compliance? The experience of a small, rural Canadian hospital. J Hum Lact 2000, 16(4):309-18.

112. Pugh LC, Serwint JR, Frick KD, Nanda JP, Sharps PW, Spatz DL, et al: A randomized controlled community-based trial to improve breastfeeding rates among urban low-income mothers. Acad Pediatr 2010, 10:14-20.

113. Quinlivan JA, Box H, Evans SF: Postnatal home visits in teenage mothers: a randomised controlled trial. Lancet 2003, 361(9361):893-900, Epub 2003/ 03/22. doi: S0140-6736(03)12770-5 [pii] 10.1016/S0140-6736(03)12770-5. PubMed PMID: 12648967

114. Redman S, Watkins J, Evans L, LLoyd D: Evaluation of an Australian intervention to encourage breast feeding in primiparous women. Health Prom Int 1995, 10(2):101-13.

115. Reifsnider E, Eckhart D: Prenatal breastfeeding education: its effect on breastfeeding among WIC participants. Journal of Human Lactation 1997, 13(2):121-5.

116. Rosen IM, Krueger MV, Carney LM, Graham JA: Prenatal breastfeeding education and breastfeeding outcomes. MCN Am J Matern Child Nurs 2008, 33(5):315-319.

117. Rossiter JC: The effect of a culture-specific education program to promote breastfeeding among Vietnamese women in Sydney. International journal of nursing studies 1994, 31(4):369-79.

118. Schafer E, Vogel MK, Viegas S, Hausafus C: Volunteer peer counselors increase breastfeeding duration among rural low-income women. Birth 1998, 25(2):101-6.

119. Schlickau J, Wilson M: Development and testing of a prenatal breastfeeding education intervention for Hispanic women. The Journal of perinatal education 2005, 14(4):24.

120. Serwint J, Wilson M, Vogelhut J, Repke J, Seidel H: A randomized controlled trial of prenatal pediatric visits for urban, low-income families. Pediatrics 1996, 98(6 Pt 1):1069-75.

121. Shaw $E$, Kaczorowski J: The effect of a peer counseling program on breastfeeding initiation and longevity in a low-income rural population. Journal of Human Lactation 1999, 15(1):19-25.

122. Taddei J, Westphal MF, Venancio S, Bogus C, Souza S: Breastfeeding training for health professionals and resultant changes in breastfeeding duration. Sao Paulo Med J 2000, 118(6):185-91.

123. Wilhelm SL, Rodehorst TK, Stepans MBF, Hertzog M, Berens C: Influence of intention and self-efficacy levels on duration of breastfeeding for midwest rural mothers. Applied Nursing Research 2008, 21(3):123-30.

124. Wolfberg AJ, Michels KB, Shields W, O'Campo P, Bronner Y, Bienstock J: Dads as breastfeeding advocates: results from a randomized controlled trial of an educational intervention. American Journal of Obstetrics and Gynecology 2004, 191(3):708-12.

125. MacArthur C, Jolly K, Ingram L, Freemantle N, Dennis CL, Hamburger R, et al: Antenatal peer support workers and initiation of breast feeding: cluster randomised controlled trial. BMJ 2009, 338:b131.

126. Pobocik RS, Benavente JC, Schwab AC, Boudreau N, Morris CH, Sue Houston M: Effect of a breastfeeding education and support program on breastfeeding initiation and duration in a culturally diverse group of adolescents. Journal of Nutrition Education 2000, 32(3):139-45.

127. Wambach KA, Aaronson L, Breedlove G, Domian EW, Rojjanasrirat W, Yeh HW: A randomized controlled trial of breastfeeding support and education for adolescent mothers. West J Nurs Res 2011, 33(4):486-505.

128. Labarere J, Gelbert-Baudino N, Laborde L, Arragain D, Schelstraete C, Francois $\mathrm{P}: \mathrm{CD}-\mathrm{ROM}$-based program for breastfeeding mothers. Maternal and Child Nutrition 2011, 7:263-72.

129. Grossman LK, Harter C, Sachs L, Kay A: The effect of postpartum lactation counseling on the duration of breast-feeding in low-income women. Am J Dis Child 1990, 144(4):471-4, Epub 1990/04/01. PubMed PMID: 2321612

130. Henderson A, Stamp G, Pincombe J: Postpartum positioning and attachment education for increasing breastfeeding: a randomized trial. Birth 2001, 28(4):236-42, Epub 2002/03/21. doi: 236 [pii]. PubMed PMID: 11903211

doi:10.1186/1471-2458-13-S3-S20

Cite this article as: Haroon et al.: Breastfeeding promotion interventions and breastfeeding practices: a systematic review. BMC Public Health 2013 13(Suppl 3):S20.

\section{Submit your next manuscript to BioMed Central and take full advantage of:}

- Convenient online submission

- Thorough peer review

- No space constraints or color figure charges

- Immediate publication on acceptance

- Inclusion in PubMed, CAS, Scopus and Google Scholar

- Research which is freely available for redistribution

Submit your manuscript at www.biomedcentral.com/submit 